\title{
Como anda o nosso relacionamento? Adaptação e validação da escala de relacionamento numa Boutique
}

How is our relationship? Adaptation and validation of partnership scale in a boutique

Larissa Costa Amuy larissa.amuy@hotmail.com Jussara Goulart da Silva - profadmjussara.ufu@gmail.com Leandro Divino Miranda Oliveira - leandro-miranda92@hotmail.com Sérgio Mendes Dutra - serggiomendes@gmail.com

\section{RESUMO}

Este trabalho enseja apresentara aplicação e a validação da Escala de Relacionamento com o Cliente (ERC) em uma boutique especializada em roupas e calçados femininos.A pesquisa, de natureza multimétodo, utilizou-se das técnicas multivariadas de análise fatorial exploratória e confirmatória. Os resultados obtidos apresentam que a Escala de Relacionamento com o Cliente (ERC) unifatorial, explicando cerca de $65 \%$ da variância e com confiabilidade de 0,788 (alfa de Cronbach), que realça a significativa correlação entre a satisfação e o relacionamento entre cliente e a boutique, com bons índices psicométricos.

Palavras-chave: Marketing de Relacionamento, CRM, Escala ERC.

\section{ABSTRACT}

This work entails presenting the construction and validation of Relationship Scale with the Client (ERC) in a specialized boutique clothing and women's shoes. The research, multi-method nature, we used multivariate techniques of exploratory and confirmatory factor analysis. The obtained results show that the Customer Relationship Scale (ERC) unifactorial, explaining about $65 \%$ of the variance and reliability of 0.788 (Cronbach alfa), which highlights the significant correlation between satisfaction and the relationship between client and boutique, with good psychometric indices.

Keywords: Relationship Marketing, CRM, ERC Scale 
INTRODUÇÃO 
Nos estudos organizacionais, a perspectiva pela busca de diferenciais competitivos, levaas empresas a seguimentarem e a estreitar seus relacionamentos com os clientes.

Atualmente, pode-se afirmar que a competitividade torna os consumidores mais exigentes, porque acarreta em diversas alternativas de consumo, o que obriga o empenho das empresas a garantir a melhor oferta para o cliente (Demo \& Rozzett, 2013).

Empresas que entregam produtos ou serviços de alta qualidade aos seus consumidores possuem grande chance de fidelizá-los, pois agregarão um valor positivo aos produtos e serviços oferecidos (Lovelock\& Wright, 2007). Portanto, o CRM (Customer Relationship Management) destacou-se em um sentido mais amplo do que ser somente um banco de dados de clientes e tornou-se suporte de informações e registo dos contatos efetuados com os clientes, por todas as áreas de negócio e de suporte, permitindo uma visão integrada e global do relacionamento com o Cliente (Payne, 2006).

O presente estudo tem como objetivo geral a aplicação e a validaçãoda Escala de Relacionamento com o Cliente (ERC) em uma boutique especializada em roupas e calçados femininos.

Este estudo se justifica pela importância atual da competitividade existente dentro deste seguimento e das mudanças do comportamento do consumidor, principalmente na década de noventa com a expansão dos PROCONS e aplicação do Código de Defesa do Consumidor. A fidelização dos clientes deste segmento tornou-se importante por meio do atendimento e estratégias de marketing.

Esta pesquisa consta de cinco seções, a parte em que se apresentou a introdução, a segunda a fundamentação teórica, em seguida os procedimentos metodológicos, posteriormente análise dos dados, e, na última seção, as conclusões e proposições referente ao estudo.

\section{FUNDAMENTAÇÃO TEÓRICA}


Esta seção apresenta o arcabouço teórico que dará sustentação à pesquisa, começando com a explanação sobre a importância do estudo do marketing de relacionamento; depois o estudo do CRM com o intuito de entender às necessidades dos clientes da empresa. Em seguida, há uma explicação da Escala de Relacionamento com o Cliente (ERC) que busca verificar o comportamento deste em relação à empresa.

\section{MARKETING DE RELACIONAMENTO}

Diante de um mercado competitivo e complexo as empresas criam estratégias de marketing com variedades e alternativas de produtos e serviços. Segundo Vavra (1993), precursor da proposta de marketing de relacionamento, definiu um composto de marketing que engloba: produto/serviço (qualidade, credibilidade, características, preço, praça, promoção, comunicação com o cliente, satisfação do cliente e serviços (serviço pré-venda e pós-venda).

O Marketing de Relacionamento constitui-se em uma ferramenta de gestão de relacionamentos utilizada pelas empresas a fim de que as mesmas tenham sucesso na fidelização de seus clientes e na rápida aceitação de novos produtos e serviços que são lançados diariamente no mercado (Golveia\& Da Rosa, 2011).

Há cinco níveis que distinguem o investimento no marketing de relacionamento. O nível básico, entendido como as necessidades primárias demandadas pelos clientes; o nível reativo, que demonstra que o empenho realizado pela empresa para ofertar o produto é o mesmo ou maior do que a concorrência; o nível responsável, que atende respeitando os direitos do consumidor; o nível proativo, que se refere a antecipar à concorrência e busca oferecer aos clientes serviços não esperados; e o nível de parceria, que procura um relacionamento de cumplicidade com os clientes de forma a agradá-los sempre (Cobra, 2009). 
Os cinco níveis mencionados por esses autores descrevem que o cliente é fundamental para as organizações, uma vez que as mesmas devem ter uma preocupação constante com a satisfação do mesmo, visto que as empresas não simplesmente vendem os produtos e/ou serviços, mas também esclarecem dúvidas sobre o produto e verificam as expectativas dos clientes. Os autores ainda afirmam que as organizações fazem parcerias com seus clientes e fornecedores, pois acreditam que o relacionamento existente entre eles torna-se um fator importante para sobreviverem em um mercado cada vez mais competitivo.

Kotler e Keller (2006) afirmam que o objetivo do marketing de relacionamento é a manutenção de uma base de clientes rentáveis que visa instrumentalizar e monitorar toda a interação entre as empresas e estes clientes.

Para Peppers e Rogers (2004), o relacionamento fundamenta-se na interação entre o cliente e a organização fornecedora. Por isso, não podemos esquecer que esse diálogo deve considerar os contatos anteriores, de modo que a cada novo encontro seja retomado onde parou, independente da época em que ocorreu.Segundo esses autores, esse é "o único modo de um relacionamento construir um contexto - que, quanto mais rico e complexo, mais vantajoso será para o cliente" (Peppers\&Rogers, 2004, p. 45).Isto é, quanto mais o vendedor lembrar detalhes sobre o comprador, quanto mais o comprador houver ensinado a seu respeito, menos vulnerável ficará o cliente aos apelos da concorrência.

Estudos realizados por Asiah, Aziz e Nazri (2011) afirmam que é fundamental que as empresas desenvolvam um relacionamento de fidelização positivo, através da compreensão das necessidades e dos desejos de seus clientes, sobretudo; o que, na visão dos autores constitui um importante diferencial. Os autores afirmam que os programas de fidelização ocasionam impactos positivos no longo prazo somente se as experiências que os clientes tiverem com as empresas forem satisfatórias.Desse modo, se faz 
necessário que ocorra um comprometimento entre todas as áreas da empresa com o programa de fidelização.Acerca dos conceitos principais de marketing de relacionamento, torna-se importante a construção da fidelização dos clientes externos através de produtos de qualidade e que lhes ofereçam benefícios. Sendo assim, a oferta de produtos e serviços torna-se um diferencial determinante da vantagem competitiva em relação a outras empresas (Kotler\& Keller, 2006) Zeithamlet al. (2013).

Diante o exposto, abordou-se neste estudo o entendimento e a administração do relacionamento entre uma empresa e seus clientes, atuais e potenciais, a fim de estabelecer relacionamentos duradouros. Diante disso, será enfocado, no próximo tópico deste estudo, o estudo do Customer Relationship Management (CRM).

\section{CUSTOMER RELATIONSHIP MANAGEMENT (CRM)}

As empresas buscam criar iniciativas de marketing relacional e aprimorar seus processos internos para oferecer um relacionamento personalizado aos seus clientes (Peppers \& Rogers, 2004). Como consequência, as pequenas e grandes empresas preocupam-se em investir no Customer Relationship Management (CRM), proficiente diferencial competitivo (Berry, 2003; Beasty, 2005).

O CRM ou Marketing de Relacionamento engloba aspectos da satisfação e da lealdade de clientes em conjunto com a constante oferta de experiências únicas e encantadoras e apresenta-se como diferencial essencial quando a questão é competitividade (Demo, 2013).

Rozzett e Demo (2010) afirmam que o CRM é um dos estudos estratégicos que se tornou uma proposta de patente relevância. Contudo, a compreensão do que o CRM significa ainda se faz limitada. Existem muitas empresas que confundem a gestão do relacionamento com o cliente com os sistemas de apoio de implementação do CRM como sendo apenas uma solução tecnológica dentro da empresa. 
Pelo fato de ser uma abordagem de um fenômeno eficaz e estratégico, o conceito de CRM adquire significados diferentes para cada autor (Winer, 2001; Chalmeta, 2005). No entanto, independentemente do que for designado, CRM é uma prática de gestão com enfoque nos clientes (Buttle, 2009). A razão para isso é que a criação de um banco de dados de clientes permite um marketing mais segmentado e com iniciativas promocionais.

Buttle (2009) menciona que o CRM pode ser analisado sob três diferentes óticas: estratégica, operacional e analítica. A perspectiva estratégica é definida pelo fato do CRM ser alocado no centro da estratégia da empresa, com o objetivo de verificar e manter clientes, criando valores positivos no longo prazo. Já, analisado pela ótica operacional, destaca a automatização das funções da empresa, isto é, a aplicação de sistemas e tecnologias de informação aos serviços, força de vendas e marketing. Por fim, a perspectiva analítica do CRM refere-se à aplicação da data mining, ou seja, coleta dados junto dos clientes que, após análise, servirão para procurar padrões que possam gerar vantagens competitivas, através da segmentação de mercados (Buttle, 2009).

Payne (2006); Loveloch e Wirtz (2007) citam que o objetivo do CRM é propiciar e cultivar um relacionamento de longo prazo com seus clientes e melhorar a eficácia e a eficiência no que diz respeito à gestão dos relacionamentos da empresa.

Tofoli, Tofoli e Santos (2006) afirmam que o uso da ferramenta CRM visa o aumento dos lucros, que o próprio CRM possibilita uma redução de custos, já que o fato de a empresa conhecer melhor seus clientes efetivos e potenciais permite direcionar, de forma mais específica e eficiente, as estratégias para a retenção dos mesmos.

No que corresponde à medida de CRM, foram encontrados alguns estudos com validação de escala baseados principalmente nos trabalhos de Wilson e Vlosky (1997) para o mercado corporativo e posteriormente, validada e adaptada ao contexto brasileiro. Viana, Cunha Jr e Slongo (2005) 
adaptaram-na para o setor industrial, que fora traduzido e pré-testado antes de sua aplicação. Foi realizada a validação fatorial exploratória e o estudo obteve sucesso na adaptação da escala, que mostrou bons índices psicométricos.

A escala de Sin,Tse e Yim (2005), pioneira na avaliação da pratica de CRM por parte da empresa, é composta por dezoito itens, divididos em quatro fatores, a saber: foco em clientes chave, organização do Customer Relationship Management, gerenciamento de conhecimentos e praticas aplicadas pelas empresas do setor de serviços financeiros de Hong Kong.

A partir daí, Soch e Sandhu (2008) desenvolveram uma escala de CRM aplicada às indústrias manufatureiras na Índia, Wang e Feng (2008) validaram uma escala de CRM para o setor de serviços na China e Öztaysi, Sezgin e Özok (2011) propuseram um instrumento para avaliar os processos internos de CRM na Turquia. AgariyaeSingh (2012a, 2012b) desenvolveram um indicador de CRM para os setores bancário e de seguros e uma escala de CRM para os clientes de hospitais públicos na Índia, e Zulkifli e Tahir (2012) validaram uma escala de práticas de CRM especificamente para clientes de bancos.

Por fim, foi realizada a partir dos estudos de Demo, Guanabara (2015) uma revisão da produção nacional acerca do CRM no período de 2009 a 2014 em 54 periódicos científicos da área de administração classificados com menção superior ou igual a B2 pelo sistema Qualis da CAPES. Os resultados evidenciaram uma produção de 35 artigos em 21 dos periódicos selecionados. Os anos de 2009 e 2012 apresentaram maior registro de artigos, correspondendo a mais de $63 \%$ da produção dos últimos 5 anos. A maioria absoluta desses artigos tratava-se de estudos teórico-empíricos, quantitativos e focavam em empresas privadas.

\section{ESCALA DE RELACIONAMENTO COM O CONSUMIDOR (ERC)}

Demo et al (2011), apresentou no Brasil estudo relacionado ao CRM, com o objetivo de "desenhar um panorama de estudos sobre CRM, apresentando 
os resultados de uma revisão bibliográfica que abrange sínteses do estado da arte e dos estudos empíricos engendrados em nível nacional no período compreendido entre 2001 e 2010".

Rozzett e Demo (2010, 2011) construíram e validaram a ERC (Escala de Relacionamento com o Cliente), utilizando a análise fatorial exploratória e confirmatória para avaliar o relacionamento entre consumidores e empresas, resultando em uma escala unifatorial com 8 itens, variância explicada superior a $64 \%$ e confiabilidade de $92 \%$ (alfa de Cronbach).

Estudos realizados por Lopes e Demo (2011) tiveram o intuito de validar duas Escalas de Relacionamento com Clientes (ERC), chamadas ERC Skol e ERC Guaraná Antarctica, buscando avaliar a percepção de relacionamento no caso específico dos consumidores da cerveja Skol e do refrigerante Guaraná Antarctica. Foi identificada a percepção dos consumidores acerca dos aspectos do seu relacionamento com as marcas, com ênfase para as diferenças de percepção entre homens e mulheres. A pesquisa multimétodo e a Análise Fatorial Exploratória para a validação experimental do instrumento, teve uma amostragem com605 sujeitos, de ambos os sexos, com idade entre 18 a 30 anos. Os resultados mostram que as ERCs Skol e Guaraná Antarctica apresentaram estrutura unifatorial com bons índices psicométricos.

Em outro estudo realizado por Vasconcelos e Demo (2011) na validação de uma Escala de Relacionamento com o Cliente (ERC) para parques temáticos, chamada ERC Parques da Disney, construída de forma a permitir a identificação da percepção do relacionamento dos turistas brasileiros com os parques do Walt Disney World, foram identificadas as percepções dos consumidores em três variáveis de controle distintas: sexo, faixa etária e número de visitas. Com uma abordagem multimétodo, utilizou-se da Análise Fatorial Exploratória para validação do instrumento com uma amostra de 391 sujeitos, cobrindo ambos os sexos, todas as faixas etárias e número de visitas previamente estipulado e; análise das médias e das variâncias (ANOVA). Na ANOVA, quando analisada a faixa etária, nota-se uma diferença de 
comportamento do público quando se avalia as conveniências (carrinhos de lanches/refeições) dos parques. Já na variável "sexo", notou-se que há uma maior percepção dos esforços da empresa em estimular a interação entre seus clientes nos turistas do sexo feminino. Assim, a ERC Parques da Disney apresenta-se como um medidor eficaz tanto para a Walt Disney Co., quanto para outros parques temáticos.

Estudos de Bateli e Demo (2012) validaram uma Escala de Relacionamento com Clientes (ERC) de Jogos do Nintendo Wii. Na etapa qualitativa, foram entrevistados 40 jogadores de diversas faixas etárias, que responderam perguntas que buscaram identificar quais os elementos mais importantes de um jogo que levam à satisfação e à fidelização do jogador. 0 pesquisador da amostra percebeu o relacionamento dos jogadores como moderadamente positivo e mostraram-se satisfeitos principalmente com a jogabilidade e o modo multijogador, dois dos conceitos centrais dos jogos do Wii e do próprio console. O instrumento obteve bons índices psicométricos, o que o torna uma importante ferramenta de diagnóstico e contribui para o aprofundamento dos estudos acerca do marketing de relacionamento.

\section{MÉTODO E TÉCNICAS DE PESQUISA}

Nesta seção estão expostos o tipo, a descrição, o cenário, a amostra, as etapas e o instrumento de pesquisa.

\section{TIPO E DESCRIÇÃO DA PESQUISA}

A pesquisa pode ser considerada como descritiva, com corte transversal e natureza multimétodo (qualitativa e quantitativa), visto que aplica dois métodos diferentes para responder a uma mesma pergunta de pesquisa (Brewer\&Hunter, 2006). Conforme os citados autores, a vantagem de realizar um estudo multimétodos reside no fato de que há convergência dos resultados encontrados, por meio de métodos diferentes, atribuindo maior credibilidade ao estudo realizado.

\section{CENÁRIO DE PESQUISA}


Atendendo aos objetivos propostos, utilizou-se como cenário de pesquisa uma boutique especializada em roupas, calçados e acessórios femininos que, por questões de sigilo, terá sua identidade resguardada. A empresa possui uma única loja situada no Pontal do Triângulo Mineiro e atende a toda a região. Seus produtos são de marcas de grife e bem conhecidas.

\section{AMOSTRA}

Realizou-se uma survey, na qual foram entrevistados os clientes da boutique que realizavam compras no dia da coleta de dados. Os clientes eram abordados no término da compra e convidados a preencherem um questionário apresentado em papel.

\section{ETAPAS DA PESQUISA}

A pesquisa está dividida em duas fases, sendo uma qualitativa e outra quantitativa. Na fase qualitativa ocorreu a adaptação e construção da escala de relacionamento desenvolvida e validada por Rozzett e Demo (2011) no cenário de pesquisa, no caso, a boutique. Para tanto, se fez necessária a realização de entrevistas com 20 clientes da boutique e a proprietária, colaborando com a compreensão objetivo proposto. A segunda fase demonstrou a confiabilidade e a validade dos itens iniciais gerados para análise por meio de análises descritivas iniciais e o uso da Análise Fatorial Exploratória - AFE, O Alfa de Cronbach. E, enfim, em seguida, encontra-se exposta a Análise Fatorial Confirmatória - AFC, com a proposta da escala de mensuração.

\section{INSTRUMENTO DE PESQUISA}

$\mathrm{Na}$ parte qualitativa para melhor entender o relacionamento da empresa com seus clientes, foi elaborado um questionário com quatro perguntas relevantes para os empreendedores posicionarem sua percepção quanto ao relacionamento da empresa com seus clientes. Assim, A boutique utiliza alguma ferramenta de fidelização do cliente? Realiza campanhas publicitárias? Quais são esses meios de divulgação? A boutique investe em 
treinamentos? O que a boutique preza de mais importante dentro da loja (atendimento, qualidade, lealdade)?

Na parte quantitativa, este trabalho trata da adaptação do questionário "Escala de Relacionamento com o Cliente - ERC elaborado e validado por Rozzett e Demo (2010)". O questionário passou por modificações, devido às dimensões observadas e coletas nas entrevistas, foi acrescido também de questões demográficas para a caracterização da amostra.

\section{PROCEDIMENTOS DA ANÁLISE DOS DADOS}

\section{FASE QUALITATIVA}

Na fase qualitativa, os dados foram colhidos através das entrevistas e houve a análise de conteúdo. Conforme Bardin (1997), a análise de conteúdo possui três fases: a pré-análise, a exploração do material e o tratamento dos resultados (inferência e interpretação). A pré-análise estabeleceu-se na preparação das perguntas, seleção dos entrevistados e a marcação das entrevistas. Para a elaboração do roteiro de entrevista, foi utilizada como base a estrutura da escala ERC, onde se pode aprofundar em questões mais direcionadas ao relacionamento com o cliente.

\section{FASE QUANTITATIVA}

Na fase quantitativa, o instrumento foi adaptado e iniciou-se a coleta de dados. Aplicação do questionário ocorreu dentro da boutique, após os consumidores terem finalizado suas compras. Os dados foram coletados e tabulados no Excel e as análises foram realizadas utilizando o software Statistical Package for the Social Sciences (SPSS) versão 21.0 e o software AMOS versão 21.0 Para fins de teste e validação da escala ERC, decidiu-se dividir a amostra em dois grupos. Com os dados do primeiro grupo, seria realizada a Análise Fatorial Exploratória (AFE) e com os dados do segundo grupo a Análise Fatorial Confirmatória (AFC).

\section{PRÉ-TESTE}


Após a elaboração de um formulário de autopreenchimento contendo as escalas já mencionadas, que deveriam ser avaliadas por meio de uma escala de 5 pontos (ancoradas em 1 - Discordo totalmente; e 5 - Concordo Totalmente), realizou-se um pré-teste para verificação de eventuais falhas ou problemas de entendimento. O resultado do pré-teste indicou que o formulário desenvolvido estava adequado para realização da coleta de dados.

\section{ANÁLISE DOS DADOS}

Nesta seção apresentam-se os resultados da pesquisa realizada, sendo dividida a caracterização da amostra em Estudo 1 e Estudo 2.

\section{CARACTERIZAÇÃO DA AMOSTRA}

Com o objetivo de testar e validar a escala de mensuração proposta neste estudo utilizou-se a mesma população composta de clientes da boutique. Foram coletados555 questionários, divididos igualmente em duas partes. No primeiro estudo, a amostra válida foi composta por 277 respondentes. Observou-se que $70,4 \%$ dos respondentes eram do sexo feminino (195 indivíduos) e 29,6\% eram do sexo masculino (82 indivíduos), demonstrando uma significativa diferença referente ao sexo dos entrevistados. Em relação à representatividade da faixa etária, 53\% dos entrevistados tinham entre 18 a 29 anos (146 indivíduos), entre 30 a 39 anos, 27\% (75 indivíduos), e acima de 40 anos (19,5\%), representando 54 indivíduos. Em relação ao tempo de cliente, 57,8\% (160 indivíduos) compravam na loja há mais de quatro anos. E há mais de um ano $41,9 \%$, perfazendo um total de 117 indivíduos.

O Critério de Classificação Econômica Brasil, enfatiza sua função de estimar o poder de compra das pessoas, abandonando a pretensão de classificar a população em termos de classes sociais. A amostra foi composta por 2,5\% dos respondentes da classe A2 (7 indivíduos) e 35,4\% da classe B1 (98 indivíduos), demonstrando uma representativa diferença em relação à classe B2, composta por $46,6 \%$ dos entrevistados (129 indivíduos); a classe C1, composta por 15,2\% (42 indivíduos) e, por fim, a classe C2, composta por apenas $4 \%$ ( 1 indivíduo). 
O sistema de pontos utilizados no Critério de Classificação Econômica Brasil é apresentado na Tabela abaixo, demonstrando a classificação econômica dos entrevistados.

Tabela 1 - Critério de Classificação Econômica Brasil.

\begin{tabular}{c|c|c}
\hline & Frequência & $\begin{array}{c}\text { Percentu } \\
\text { al }\end{array}$ \\
\hline A2 & 7 & 2,5 \\
\hline B1 & 98 & 35,4 \\
\hline B2 & 129 & 46,6 \\
\hline C1 & 42 & 15,2 \\
\hline C2 & 1 & 4 \\
\hline Total & 277 & 100 \\
\hline
\end{tabular}

Fonte: Coleta de dados SPSS 20.0.

ESTUDO 1 - ANÁLISE FATORIAL EXPLORATÓRIA 
Como planejado, dividiu-se a amostra em dois grupos, sendo que um serviu para a realização da Análise Fatorial Exploratória (AFE). Este grupo é formado por 277 questionários. Em relação à validação psicométrica, foi utilizada a Analise Fatorial Exploratória - AFE, sendo possível, por meio da Análise dos Componentes Principais, decidir quantos fatores seriam extraídos. Quanto à Análise Fatorial, foi utilizada a Análise Fatorial Exploratória (AFE), técnica utilizada na psicometria, importante quando objetiva a validação de instrumentos psicológicos (Pasquali, 2008).

Segundo Tabachnick e Fidell (2001), a AFE tem como objetivo a descrição e simplificação dos dados coletados, agrupando as variáveis que mantem correlação entre si. Hair et al (2005) enfatizam que em uma amostra de 150 casos as cargas fatoriais acima de 0,45 são significativas. Pasquali (2008) aponta que alfas acima de 0,70 são considerados confiáveis e, acima de 0,80, muito confiáveis. Já para Neiva, Abbad e Trócoli (2008), devem ser considerados índices maiores ou iguais a 0,75, apesar da literatura internacional aceitar índices superiores a 0,60. Para a aferição de confiabilidade do fator, foi utilizado índice Alfa de Cronbach, que indicou um fator bastante confiável para a escala. A ERC em uma boutique especializada em roupas e calçados femininos obteve um alfa igual a 0,835, classificando-se muito confiável.

Tabela 2 - Confiabilidade-ERC boutique especializados em roupas e calçados femininos.

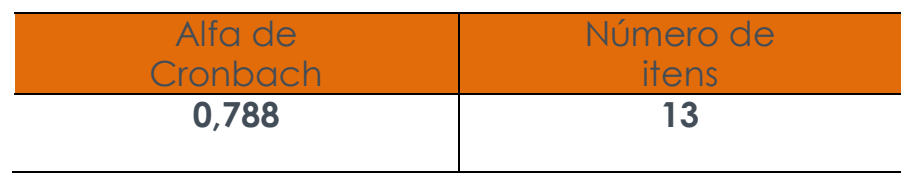

Fonte: Elaborado pelos autores.

Quanto à variância explicada, o índice foi de 65,21\%. O Alfa de Cronbach está dentro do aceitável, ou seja, os resultados são confiáveis para a validação da Escala ERC na boutique especializada em roupas e acessórios femininos. 
Em relação à fatorabilidade, analisou-se a matriz de correlações. Os tamanhos das correlações foram inspecionados e então foram observados o determinante da matriz e os resultados do teste de adequação da amostra de Kaiser-Meyer-Olkin (KMO). O valor foi 0,788, números considerados satisfatórios para a fatorabilidade da amostra.

Para a extração de fator, foi feita a Análise dos Componentes Principais ou Principal Components Analysis (PCA), que considera a variância total dos dados e procura uma entre as variáveis a fim de que um máximo de variância seja explicada por essa combinação (Dias filho, Corrar, \& Paulo, 2007). Sendo a matriz fatorável, de acordo com o teste KMO, foram examinados os eigenvalues (autovalores), a porcentagem de variância explicada de cada fator, o gráfico scree plot (gráfico de declive) e a análise paralela, a fim de determinar a quantidade de fatores a serem retirados. Depois de definir a quantidade de fatores, um dos eixos principais de análise (PAF) foi executado usando a rotação Promax - já era esperada correlação entre os fatores, o que é comum em fenômenos comportamentais.

Segundo Pasquali (2008), na situação em que as correlações entre os fatores extraídos sejam significativas (acima de 0,3), é possível identificar a presença de fatores de segunda ordem. Contudo, foram extraídos seis fatores, sendo que as questões Q3, Q4, Q8, Q19 e Q20 foram excluídas das análises posteriores por apresentarem uma carga fatorial abaixo de 0,35.

Quanto à validade desta, Pasquali (2008) ressalta que um item válido é aquele que representa bem um fator, ou seja, boa carga fatorial. Comrey (1973) menciona que classificados os itens com carga maior ou igual a 0,71 como excelentes; maior ou igual há 0,63 como muito bons; maior ou igual a 0,55 como bons; maior ou igual a 0,45 como razoáveis; maior ou igual a 0,32 como cargas fatoriais pobres.

Devido às análises terem representado um resultado satisfatório quanto aos índices psicométricos da escala, os itens da ERC apresentaram adesão aos conceitos teóricos que as apoiam. 
Tabela 3 - Índices psicométricos da ERC na boutique especializada em roupas e calçados femininos.

\begin{tabular}{|c|c|c|c|}
\hline Item & $\begin{array}{c}\text { Descriçã } \\
0\end{array}$ & Fator & $\begin{array}{l}\text { Qualidad } \\
\text { e }\end{array}$ \\
\hline Q1 & Eu compro nesta boutique regularmente & 0,754 & Excelente \\
\hline Q2 & As Experiência de compras com a boutique & 0,754 & Excelente \\
\hline Q6 & A boutique possui credibilidade & 0,761 & Excelente \\
\hline Q9 & Sou fiel ao comprar roupas e sapatos nesta boutique & 0,674 & $\begin{array}{c}\text { Muito } \\
\text { Bom }\end{array}$ \\
\hline Q10 & $\begin{array}{l}\text { A boutique realiza campanhas promocionais, desfiles, } \\
\text { eventos promovendo interação }\end{array}$ & 0,741 & Excelente \\
\hline Q11 & $\begin{array}{l}\text { A boutique não possui concorrentes que tenham a } \\
\text { mesma importância para mim }\end{array}$ & 0,646 & $\begin{array}{l}\text { Muito } \\
\text { Bom }\end{array}$ \\
\hline Q12 & $\begin{array}{l}\text { Está presente nos veículos de comunicação, e possui } \\
\text { fácil acesso às informações }\end{array}$ & 0,659 & $\begin{array}{l}\text { Muito } \\
\text { Bom }\end{array}$ \\
\hline Q13 & A boutique realiza pesquisas de mercado & 0,634 & $\begin{array}{l}\text { Muito } \\
\text { Bom }\end{array}$ \\
\hline Q14 & Confio na boutique & 0,656 & $\begin{array}{l}\text { Muito } \\
\text { Bom }\end{array}$ \\
\hline Q15 & $\begin{array}{l}\text { Eu recomendo esta boutique aos meus amigos e } \\
\text { familiares }\end{array}$ & 0,650 & $\begin{array}{c}\text { Muito } \\
\text { Bom }\end{array}$ \\
\hline Q16 & Satisfação no atendimento pessoal na boutique & 0,687 & $\begin{array}{l}\text { Muito } \\
\text { Bom }\end{array}$ \\
\hline Q17 & Satisfação no atendimento por telefone & 0,744 & Excelente \\
\hline Q18 & Satisfação no atendimento pela internet & 0,674 & $\begin{array}{l}\text { Muito } \\
\text { Bom }\end{array}$ \\
\hline
\end{tabular}

Fonte: Elaborado pelos autores.

A ERC da boutique foco deste estudo obteve cinco itens (Q1, Q2, Q6, Q10 e Q17) com classificação "excelente", dez itens (Q5, Q7, Q9, Q11, Q12, Q13, Q14, Q15, Q16 e Q18) com classificação "muito bom". A identificação do consumidor com o produto nas questões 1, 2, 10, 12 e 13 é um indicador de relacionamento validado teoricamente por Demo e Pontes (2008). Esta atitude de recomendação dentre os itens Q15, Q16 encontra-se nos trabalhos realizados por Griffin (2002), ao afirmar que clientes fidelizados não apenas repetem suas compras inúmeras vezes, mas também a defendem e recomendam para novos clientes.

Segundo Kotler (2006), é necessário que o atendente de uma empresa conheça os requisitos básicos para um bom atendimento ao cliente, e que esses clientes confiem na empresa (Q6, Q14, Q17 e Q18) revelam o valor de um cliente satisfeito para a construção e manutenção de um 
relacionamento, conforme menciona Vavra (1993). O consumo regular do produto (Q9) é validado pelo autor Cobra (2009), que corrobora que a frequência de compra pode ser capaz de apontar a fidelização de clientes e consequência disso à manutenção de um relacionamento. Peppers e Rogers (2004) afirmam que se lembrar dos detalhes dos compradores e conhecer os gostos e desejos dos compradores, menos vulneráveis a concorrência serão (Q11). Foi utilizada a escala tipo de Likert de 5 pontos para avaliar a percepção dos clientes pesquisados quanto aos aspectos de relacionamento com a ERC em uma boutique especializada em roupas e calçados femininos.

\section{ESTUDO 2 - ANÁLISE FATORIAL CONFIRMATÓRIA}

Para esta fase, utilizou-se o segundo grupo amostral, formado pelos outros 277 respondentes.Segundo Malhotra (2012) e Hair Jr.et al (2009), a Análise Fatorial Confirmatória é um caso especial de uma técnica mais ampla, denominada modelagem de equações estruturais.

Foi mensurada a análise confirmatória por meio da medida da confiabilidade. Chin (1998), propõe, uma medida de confiabilidade melhor do que o alfa de Cronbach em equações estruturais do modelo, dado que se baseia na análise das cargas, ao invés de as correlações entre as variáveis observadas. A validade de constructo foi examinada neste estudo através de validade convergente. Hair et al (2009).

A estrutura que se adequou melhor na ERC utilizada na boutique especializada em roupas e calçados foi o software AMOS versão 21.0, que extraiu os seguintes índices: $N C=5,485(P<0,001)$ qui-quadrado sobre graus de liberdade (no output como CMIN/DF): divide-se o valor do qui-quadrado pelo número de grau de liberdade para obter um valor de ajuste ao modelo menos sensível ao tamanho. Valores menores que 3 são preferíveis, mas valores abaixo de 5 são toleráveis (Bryne, 2001). O CFI= 0,98 (índice de ajuste comparativo): índice similar ao NFl, que faz uso de uma distribuição de quiquadrado não-central, e que procura levar em consideração a 
complexidade de um modelo. Idealmente, valores maiores que 0,95 também são desejados (Thompson, 2004). A vantagem do uso do CFI é evitar a subestimação do ajuste observado no NFI quando a amostra é pequena (Thompson, 2004). O RMSEA= 0,070 (raiz da média dos quadrados dos erros de aproximação): ao contrário do RMR, O RMSEA possui uma distribuição conhecida e, portanto, representa de forma mais adequada quão bem um modelo se ajusta á população, não apenas à amostra utilizada para a estimação. Thompson (2004) considera que valores abaixo de 0,05 são considerados ótimos e os valores acima de 0,06 geralmente indicam um ajuste razoável para o modelo.

Em suma, os resultados são animadores em termos de generalização escala. Os 15 itens da escala ERC propostos neste estudo foram encontrados para ter um alto grau de confiabilidade e validade e, por isso, podem ser usados para entender corretamente quais aspectos são considerados relevantes na sua relação com as empresas e, em geral, que podem influenciar suas experiências de compras e satisfação.

O resultado desta análise indicou que a ERC aplicada na boutique especializada em roupas e calçados femininos é um construto unidimensional. É importante ressaltar que, na análise confirmatória, incialmente aplicado na mesma estrutura foram expurgados seis itens devido as análises apresentarem uma carga fatorial abaixo de 0,35, de acordo com a avaliação literatura e com a validação exploratória. Por último, os resultados originados confirmaram a validação Escala ERC, mostrando a adequação conceitual da estrutura obtida na análise exploratória e em forma satisfatória.

Além disso, todos os 15 itens foram significativos, carregados como previsto no seu fator. Estes resultados confirmam a evidência para sugerir que a escala proposta validado neste estudo é uma medida operacional confiável para boutique especializada em roupas e calçados femininos.

\section{CONSIDERAÇÕES FINAIS}


O presente estudo teve como objetivo geral a aplicação e a validação da escala de relacionamento com o cliente (ERC) em uma boutique especializada em roupas e calçados femininos. Observou- se que a boutique tem um relacionamento consistente com os seus clientes. Quando realizada a análise de conteúdo na etapa qualitativa, os resultados foram satisfatórios com maior frequência nas entrevistas, que acabaram por dar suporte a questões da versão da ERC. Em relação à pesquisa quantitativa, foi realizada a Análise Fatorial Exploratória, que contribuiu para a medição da percepção dos aspectos do relacionamento. A Análise Fatorial Confirmatória apresentou índices psicométricos relevantes para a validação do instrumento.

\section{IMPLICAÇÕES ACADÊMICAS}

Destaca-se como principal contribuição acadêmica deste trabalho o desenvolvimento de uma estrutura teórica de relacionamentos causais entre variáveis latentes de Marketing de Relacionamento e oferta de serviços ao cliente.

Recomenda-se a realização de estudos em futuras pesquisas acadêmicas que avaliem a perspectiva da empresa acerca do relacionamento para verificar se realmente há esforços em fidelizar o cliente e construir um relacionamento com ele.

\section{IMPLICAÇÕES GERENCIAIS}

Vale ressaltar que a escala criada e validada constitui uma importante contribuição empresarial, haja vista que a Administração é uma ciência essencialmente aplicada, porque ressalta a importância do relacionamento da empresa com o cliente. Além disso, a escala criada mostrou-se um instrumento de alta qualidade, segundo os índices psicométricos obtidos, e constitui uma ótima ferramenta de avaliação para a boutique.

\section{PRINCIPAIS LIMITAÇÕES E SUGESTÕES PARA ESTUDOS FUTUROS}


O objetivo geral desta pesquisa foi atingido: aplicar e validar a escala, a partir da ERC validada por Rozzett e Demo (2010), para avaliar a percepção dos clientes quanto ao relacionamento com a empresa.

É necessário que haja mais estudos sobre este tema. Sugerem-se novas validações, pois os únicos trabalhos encontrados foram realizados por Rozzett e Demo (2010); há poucas publicações de ensaios teóricos sobre Marketing de relacionamento, bem como estudos com natureza multimétodo.

Uma das limitações em relação ao instrumento (questionário) de coleta de dados é sua sujeição a distorções nos dados coletados, devido à dependência da interpretação do respondente. Destacando também que nem todos os clientes veem a importância da colaboração de uma pesquisa, pois se recusam de fazerem parte dela.

Por fim, considerando a importância da construção das estratégias de relacionamento, a ERC na boutique constituiu um importante instrumento diagnóstico, tanto para a boutique foco de estudo quanto para outras boutiques que pretendem ou já implementaram estratégias de fidelização com o cliente.

\section{REFERÊNCIAS BIBLIOGRÁFICAS}

Agariya, A. K. \& Singh, D.(2012). CRM Index development and validation in Indian banking sector. International Journal of Customer Relationship Marketing and Management, 3(2), 10-32.

Agariya,A. K.; Singh, D.(2012). CRM Scale development \& validation in Indian insurance sector. Journal of Internet Banking and Commerce, 17 (2), 1$21,2012 a$.

Agariya, A. K.; Singh, D.(2013). CRM scale development and validation scale in Indian public hospitals. Journal of Health Management, 15(2), 275$291,2013$.

Alvarenga, C. R. F. M. (2013). O Marketing de Relacionamento como ferramenta na fidelização do cliente: um estudo no armazém Paraíba- 
Campina Grande. 2013. 28 f. Artigo (Bacharelado em Administração) Centro de Ciências Exatas e Sociais Aplicadas, Universidade estadual da Paraíba, Patos-PB.

Asiah, O., Azis N. \& Nazri, M. Understanding the relat relationship of program satisfaction, program loyalty and store loyalty among cardholders of loyalty programs. Asian Academy of Management Journal. V. 16, No.1, p.21$41,2011$.

Bardin, L.(1997). Análise de conteúdo. Lisboa: Edições 70.

Batelli, L. \& Demo G. (2012). Desenvolvimento e Validação de uma Escala para avaliar o Relacionamento entre jogadores e jogos do Nintendo WII. Universidade de Brasília, Brasília. Disponível em:

http://www.abepro.org.br/biblioteca/ENEGEP2012_TN_STP_163_952_20604.pd. Acesso em 21 jan. 2015.

Beasty, C. (2005). Customer Relationship Management Magazine, v. 9, n. 8, p.19, Aug.

Berry, D. (2003). CRM for the small to medium enterprise--king-sized CRM on a bite-sized budget. Customer Inter@ction Solutions, v. 2, n. 11, p. 56-59.

Brewer, J. \& Hunter, A.(2006). Foundations of Multimethod Research. Sage, Thousand Oaks.

Bryne, B. (2001). Structural equation modeling with Amos: Basic concepts, applications and programming. New Jersey: Lawrence Erlbaum Associates.

Buttle, F.(2009). Customer Relationship Management, Burlington, MA: Elsevier.

Carvalho, L. R. B.(2013). Determinantes que influenciam a satisfação e fidelização de clientes no setor de distribuição de produtos de higiene e limpeza profissional. 160 f. Artigo (Mestrado em Comunicação e Marketing). Instituto Politécnico de Viseu, Escola Superior de educação de Viseu. 
Chalmeta, R. (2005). Methodology for customer relationship management, Grupo integracion Y Re-ingenieria de Sistemas (IRIS), Universitat Jaume I, The Journal of Systems and Software, 79; p.1015-1024.

Chin, W. W. (1998). The partial least squares approach to structural equation modeling. In G. A. Marcoulides (Ed.), Methodology for business and management. Modern methods for business research (pp. 295-336). Mahwah, NJ, US: Lawrence Erlbaum Associates Publishers.

Cobra, M. (2009). Administração de marketing no Brasil. 3. ed. Rio de Janeiro: Elsevier.

Comrey, A.L. (1973). A first course in factor analysis. New York: Academic Press.

Demo P. \& Ponte V.(2008). Marketing de relacionamento (CRM): estado da arte e estudo de casos. São Paulo: Atlas.

Demo, G. \& Guanabara, M. (2015). Marketing de Relacionamento com a Apple: O papel do julgamento e significado de produto na escolha de iPHONE. Read. Porto Alegre.ed. 80 No 1 -p. 170-197.

Demo, G. (2014). B2C Market: Development of a CRM Scale. In: Ali Ghorbani. (Ed.). Marketing in the Cyber Era. Hershey, PA: IGI Global, p. 85-95.

Dias Filho, J. M, Corrar, L. J, \& Paulo, E. (2007). Análise multivariada. São Paulo: Atlas.

Figueiredo F. D. B. \&Silva J. J.A. (2010). Visão além do alcance: uma introdução à análise fatorial.Opin. Pública, Campinas, v.16, n.1, June.Available from <http://www.scielo.br/pdf/op/v16n1/a07v16n1>. Acesso em: 06 jan.2015.

Golveia, F. J. P.\& Da Rosa, M. W. B. (2011). A importância do marketing de relacionamento para as Organizações: foco no cliente externo. Revista científica eletrônica de ciências sociais aplicadas da EDUVALE. Publicação científica da Faculdade de Ciências Sociais aplicadas do Vale de São Lourenço-Jaciara/MT. Ano IV, Número 06, novembro. Disponível 
em:<http://www.eduvalesl.edu.br/site/edicao/edicao-39.pdf>. Acesso em: 03 ag. 2014.

Griffin, J. (2002). Como conquistar e manter o cliente fiel: transforme seus clientes em verdadeiros parceiros. São Paulo: Futura.

HAIR, J. F. JR. et al. (2009)Análise Multivariada de Dados. 6. ed. Porto Alegre: Bookman.

Hair, Jr. e J. F. et al. (2005). Fundamentos de métodos de pesquisas em administração. Porto Alegre: Bookman.

Hora, H., Monteiro, G. \&Arica, J. (2010). Confiabilidade em Questionários para Qualidade: Um estudo com o coeficiente Alfa de Cronbach. Disponível em: <http://seer.ufrgs.br/ProdutoProducao/article/view/9321>. Acesso em 21 jan. 2015.

Kotler P. \& Keller, K. L. (2006). Administração de Marketing: A Bíblia do Marketing. 12. ed. São Paulo: Pearson / Prentice-Hall.

Kotler, P. (2006). Administração de marketing. 10. ed. São Paulo: PrenticeHall.

Lopes, C. \& Demo (2012).Desenvolvimento e Validação das Escalas de Relacionamento com clientes da cerveja Skol e guaraná Antártica. Universidade de Brasília. Disponível em:<http://www.anpad.org.br/diversos/trabalhos/EMA/ema_2012/2012_EMA 9.pdf.>. Acesso em: 21 jan. 2015.

Lopes, C. \& Demo G. (2011). Desenvolvimento e Validação das Escalas de Relacionamento com clientes da cerveja skol e guaraná antártica. Universidade Federal de Brasília. Brasília.

Lovelock, C \&Wirtz, J.(2007). Services Marketing: people, technology, strategy. 6th. ed. Upper Saddle River: Prentice Hall.

Malhotra\& Naresh K. (2012). Pesquisa de Marketing: Uma orientação aplicada. 6. ed. Porto Alegre: Bookman. 
Marcoulides, G. A. (1998). Modern Methods for Business Research. Mahwah, NJ: Lawrence Erlbaum Associates, p. 295-358.

Neiva, E. R, Abbad, G \& Tróccoli, B. T. (2008). Roteiro para análise fatorial de dados. Brasília: [S.n.], (Apostila da disciplina Análises Multivariadas e Regressão Múltipla, Programa de Pós-Graduação em Psicologia Social, do Trabalho e das Organizações, Instituto de Psicologia, Universidade de Brasília).

Öztaysl, B., Sezgin, S. \& Özok, A. F. (2011). A measurement tool for customer relationship management processes. Industrial Management \& Data Systems, $111(6)$, 943-960.

Pasquali, L. (2008). Análise fatorial para pesquisadores. Laboratório de Pesquisa em Avaliação e Medida (LabPAM) - Instituto de Psicologia. Brasília: Universidade de Brasília.

Payne, A.(2006).Handbook of CRM: achieving excellence in customer relationship management. Oxford: Elsevier.

Peppers, D.\& Rogers, M. (2004). Marketing um a um marketing individualizado na era do cliente. 2. ed. Rio de Janeiro: Campus.

Richardson, R. J. (2011). Pesquisa Social: Métodos e Técnicas. São Paulo: Atlas.

Rozzet, K. \& Demo G. (2013). Customer Relationship Management Scale for the Business-toConsumer Market: Exploratory and Confirmatory Validation and Models Comparison. International Business Research. Canadá, p. 29-42. Set.

Rozzet, K. \& Demo, G(2010). Desenvolvimento e validação da Escala de Relacionamento com Clientes (ERC). Revista Administração de Empresas, 50(4), 383-395.

Rozzet, K. \& Demo, G. (2010). Desenvolvimento e validação da Escala de Relacionamento com Clientes (ERC). Encontro Nacional de Engenharia de Produção, São Carlos, SP, Brazil, Outubro. 
Rozzet, K. \& Demo, G. (2010). Desenvolvimento e validação fatorial da escala de relacionamento com clientes (ERC). RAE. São Paulo. v. 50. n. 4, out./dez.

Rozzet, K. \& Demo, G.(2011). Escala de Relacionamento com Clientes (ERC): validação fatorial confirmatória por meio da modelagem por equações estruturais. In Congresso Iberoamericano de Psicologia das Organizações e do Trabalho, 2. Florianópolis: SBPOT.

Rozzett, K. \&Demo, G. (2014). Desenvolvimento e validação da Escala de Relacionamento com clientes (ERC), XXX ENCONTRO NACIONAL DE ENGENHARIA DE PRODUÇÃO, São Carlos, p. 14.

Sin, L. Y. M, Tse, A. C. B \& Yim, F. H. K.(2005). CRM. Conceptualization and scale development. European Journal of Marketing, v. 39, n. 11/12, p. 12641290.

Soch, H., Sandhu, H. S. (2008). Does Customer Relationship Management affect firm performance? Global Business Review, 9(2), 189-206.

Tabachnick, B, Fidell, L. S.(2001). Using multivariate statistics. 4th ed. San Francisco: Allyn \& Bacon.

Thompson, B. (2004). Ten commandments of structural equation modeling. Em L. G. Grimm \& P. R. Yarnold (Orgs.), Reading and understanding more multivariate statistics (p. 261-283). Washington: American Psychological Association.

Tofoli, E. T., Tofoli, I., Santos, A. R. P. d. (2006). Estratégia do Marketing de Relacionamento em Empresa Orientada para o Mercado na Conquista de uma Maior Participação do Mercado, In: IV Simpósio de Gestão e Estratégia em Negócios, Seropédica, RJ, Brasil, Setembro.

Vasconcelo, H. \&Demo G. (2011).Encantar para fidelizar: um estudo instrumental sobre o relacionamento entre parques do Walt Disney Word e seus clientes brasileiros. Universidade de Brasília, Brasília. 
Vavra, T. (1993). Marketing de relacionamento: como manter a fidelidade de clientes através do marketing de relacionamento. São Paulo: Atlas.

Viana, D. A., Cunha JR, M. V. M., Slongo, L. A.(2005). Medindo O conceito de marketing de relacionamento no contexto brasileiro: a validação de uma escala no setor industrial. In: ENCONTRO NACIONAL DA ANPAD. Anais. Foz do Iguaçu.

Wang Y. \& Feng H. (2008) CRM capability in service industries: conceptualization and scale development. Proceedings of 2008 IEEE International Conference on Service Operations and Logistics, and Informatics, Beijing, China.

Wang, Y., Feng, H. (2008). CRM capability in service industries: conceptualization and scale development. Proceedings of 2008 IEEE International Conference on Service Operations and Logistics, and Informatics, Beijing, China.

Wilson, E. J., Vlosky, R. P. (1997). Partnering relationship activities: building theory from case study research. Journal of Business Research, v.39, n. 1, p. 5970 .

Winer, R. (2001).A Framework for Customer Relationship Management, California Management Review, 43, 4, p.89-105.

Zeithaml, V. A., Bitner, M. J., Gremler, D. D.(2013). Marketing de Serviço: A empresa com foco no cliente. 6. ed., Livro online, <http://books.google.com.br/books >. Acessado em: 10 jul.2014.

Zulkifli, Z., Tahir, I. M. (2012). Developing and validating Customer Relationship Management (CRM) practices construct. International Journal of Business and Behavioral Sciences, 2(1), 35-48. 